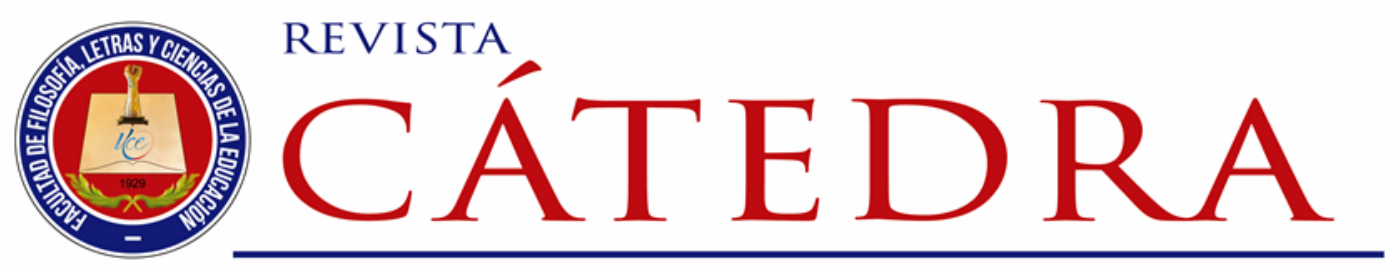

\title{
Análisis de la implementación del portal de servicios educativos: EducarEcuador
}

\section{Analysis of the implementation of the educational services portal: EducarEcuador}

\author{
Santiago Vinueza-Vinueza \\ Universidad Central del Ecuador, Quito, Ecuador \\ sfvinueza@uce.edu.ec \\ http://orcid.org/0000-0002-0818-6554
}

Joshua Pozo-Caicedo

Universidad Central del Ecuador, Quito, Ecuador jdpozo@uce.edu.ec https://orcid.org/0000-0001-9482-5369

Anthony Pacheco-Gallegos Universidad Central del Ecuador, Quito, Ecuador aspachecog@uce.edu.ec https://orcid.org/0000-0002-8908-0363

Richard Arequipa-Caisaluisa Universidad Central del Ecuador, Quito, Ecuador rsarequipa@uce.edu.ec https://orcid.org/0000-0001-5756-450X

(Recibido: 07/04/2020; Aceptado: 10/04/2020; Versión final recibida: 02/05/2020)

Cita del artículo: Vinueza-Vinueza, S. Pozo-Caicedo, J. Pacheco-Gallegos, A. y Arequipa-Caisaluisa, R. (2020). Análisis de la implementación del portal de servicios educativos: EducarEcuador. Revista Cátedra, 3(2), 99-111.

\section{Resumen}

Este artículo presenta el análisis sobre la implementación de la plataforma educativa EducarEcuador en el sistema de educación nacional, además, la importancia del uso de las

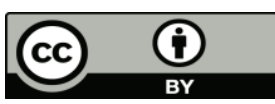

\section{Licencia Creative Commons Atribución 4.0 Internacional (CC BY 4.0)}


plataformas virtuales en la actualidad. Es necesario recalcar que existen zonas que por su ubicación geográfica y económica no disponen de acceso a tecnologías de la información y comunicación (TIC). La investigación nos permitirá conocer el estado de la implementación de la plataforma virtual EducarEcuador. Esta investigación se realizará a partir de una revisión de diferentes fuentes bibliográficas, se indagaron experiencias y resultados de investigaciones sobre la plataforma EducarEcuador. También se usaron los resultados obtenidos del análisis de documentos estadísticos del Ministerio de Educación y del Instituto Nacional de Estadísticas y Censos (INEC). Sin embargo, las investigaciones encontradas son a nivel institucional, cantonal o provincial. Lo cual no permite conocer el estado real de la implementación de la plataforma EducarEcuador en todo el ámbito educativo ecuatoriano. La investigación busca evaluar el índice de la incorporación de la plataforma virtual en las instituciones educativas del país. Entre los resultados se percibe que la implementación y el manejo de las herramientas de la plataforma ha tenido una mayor acogida en la jurisdicción fiscal. Además, se percibe la falta de infraestructura tecnológicas en el país. En consecuencia, la ausencia de infraestructura limita al manejo de la plataforma educativa. No obstante, no limita solamente a los usuarios sino limita fuertemente la implementación de esta plataforma.

\section{Palabras clave}

EducarEcuador, gestión escolar, MinEduc, servicios escolares.

\section{Abstract}

This article presents the analysis of the implementation of the EducarEcuador educational platform in the national education system, as well as the importance of using virtual platforms today. It is necessary to emphasize that there are areas that due to their geographical and economic location do not have access to information and communication technologies (ICT). The research will allow to know the status of the implementation of the virtual platform EducarEcuador. This research will be carried out from a review of different bibliographic sources, and experiences and research results on the EducarEcuador platform were investigated. The results obtained from the analysis of statistical documents of the Ministry of Education and the National Institute of Statistics and Censuses (INEC) were also used. However, the investigations found are at the institutional, cantonal or provincial level. This does not allow knowing the real status of the implementation of the EducarEcuador platform in the entire Ecuadorian educational environment. The research seeks to evaluate the rate of incorporation of the virtual platform in the country's educational institutions. Among the results, it is perceived that the implementation and management of the platform's tools has been more well received in the jurisdiction. In addition, the lack of technological infrastructure in the country is perceived. Consequently, the absence of infrastructure limits the management of the educational platform. However, it does not only limit users but it strongly limits the implementation of this platform.

\section{Keywords}

EducarEcuador, educative management, MinEduc, school services.

\section{Introducción}

El siguiente artículo pretende analizar el nivel de implementación de la plataforma educativa EducarEcuador. Dicha plataforma fue desarrollada he implementada por el Ministerio de Educación para mejorar la calidad del servicio educativo en el país. Al respecto, Cabrero (2005) dice "Estos programas en líneas generales permiten realizar una serie de actividades como las siguientes: ubicar diferentes tipos de documentos (textos, imágenes, direcciones web, presentaciones colectivas informatizadas...)" (pág. 8). Por lo cual, Las plataformas con fines educativos buscan ayudar y facilitar procesos a la comunidad educativa. Sin embargo, después de

\section{Licencia Creative Commons Atribución 4.0 Internacional (CC BY 4.0)}

Revista Cátedra, 3(2), pp. 99-111, mayo-agosto 2020. e-ISSN: 2631-2875

https://doi.org/10.29166/10.29166/catedra.v3i2.2210 
su implementación se dieron a conocer falencias dentro del funcionamiento y accesibilidad a la plataforma educativa EducarEcuador.

Para la elaboración del presente artículo la búsqueda de información ha sido uno de los principales obstáculos, puesto que la página EducarEcuador presentó errores en los servidores, lo que dificultó la extracción de la información necesaria para el artículo. Además, la escasez de fuentes bibliográficas fue un limitante puesto que, no existen numerosos estudios a cerca del análisis de la plataforma virtual en los diferentes tipos de instituciones educativas. El desafío que plantea pretende determinar el nivel de implementación de la plataforma educativa a nivel nacional, además determinar las dificultades que se presenta la comunidad educativa, los alumnos, profesores, padres de familia, al acceder a la plataforma virtual EducarEcuador, en las diferentes regiones del Ecuador.

Si se desea mejorar el proceso de enseñanza-aprendizaje a través del uso de las plataformas educativas, es fundamental conocerla a fondo. Según Taipe (2016):

La plataforma de educativa virtual ha constituido unos de los pilares fundamentales en la construcción del conocimiento, en la formación a través de internet, el aprendizaje colaborativo puede facilitarse mediante herramientas sincrónicas o asincrónicas tales como el Chat, el correo electrónico o las listas de distribución y foros (pág. 24).

Es muy importante conocer sobre sus herramientas, ya que ayudará para mejorar el proceso de enseñanza aprendizaje. También, conocer sus ventajas y desventajas que posee la utilización de la plataforma. Adicionalmentes es importante determinar los factores que influyen en la utilización tanto externa como internamente de la plataforma EducarEcuador.

Con el análisis de la plataforma de servicios educativos EducarEcuador, se pretende determinar las principales falencias de acceso para los usuarios tales como: estudiantes, padres de familia, profesores y la comunidad educativa en general. A demás de la implementación de la misma a nivel nacional. De tal forma poder llegar a un diagnóstico en el que se pueda dar una opinión objetiva de cuáles pueden ser las posibles soluciones para que la plataforma tenga tanto éxito como otros portales educativos. El Ecuador necesita una plataforma estable y amigable con el usuario para poder modernizar el sistema educativo actual por lo que dicho análisis es necesario.

La finalidad de este artículo es realizar un análisis a la plataforma de servicios educativos EducarEcuador, con el fin de identificar las funciones y servicios que brinda dicha plataforma a la comunidad educativa, además, identificar las dificultades que se presentan al acceder a la plataforma virtual EducarEcuador en las zonas rurales del país. Es necesario, determinar el comportamiento de la plataforma tanto en la costa, sierra, oriente y región insular del país, por lo que se busca dar respuesta a las siguientes preguntas:

- ¿Cuál es el nivel de implementación de las instituciones educativas por periodo lectivo a la plataforma EducarEcuador?

- ¿Cuáles son las dificultades que se presentan para la comunidad educativa en las zonas rurales al acceder a la plataforma virtual EducarEcuador?

Esta investigación se encuentra enfocada a nivel nacional, ya que la plataforma EducarEcuador es desarrollada e implementada en el sistema educativo por el Ministerio de Educación de Ecuador. Esta abarca el sistema educativo tanto al régimen costa como sierra. Las dificultades que se presentan para hacer uso de la plataforma EducarEcuador en ciertos sectores educativos como las zonas rurales podrían representar un obstáculo para el desarrollo de una educación moderna. Por lo que el propósito del artículo es mostrar cuales son las falencias que presenta la plataforma

Licencia Creative Commons Atribución 4.0 Internacional (CC BY 4.0)

Revista Cátedra, 3(2), pp. 99-111, mayo-agosto 2020. e-ISSN: 2631-2875

https://doi.org/10.29166/10.29166/catedra.v3i2.2210 
educativa del Ministerio de Educación (EducarEcuador), haciendo un análisis técnico y comparativo frente a otras plataformas educativas.

El artículo se encuentra estructurado en seis secciones. En la primera sección se hace referencia a la introducción del artículo. La segunda sección presenta los conceptos relacionados a cerca de la plataforma virtual EducarEcuador. En la tercera sección se analiza una variedad fuentes bibliográficas que presentan resultados y conclusiones relacionadas con la plataforma virtual EducarEcuador. La cuarta sección presenta la metodología que se utilizó para desarrollar el presente artículo de manera detallada. En la quinta sección se da a conocer los resultados obtenidos a cerca del análisis de la plataforma virtual EducarEcuador. Finalmente, en la sexta sección se determinan conclusiones a partir de los resultados del análisis de la plataforma educativa EducarEcuador

\section{Conceptos relacionados}

\subsection{Las TIC en la educación}

La implementación de las Tecnologías de la información y la comunicación (TIC) dentro de una sociedad de conocimientos ha causado enormes cambios. La educación es uno de los principales usuarios de las TIC y la cual ha contraído una gran mejora en el proceso de enseñanza aprendizaje. Según Hernández (2017) "uno de los lugares donde la tecnología ha influenciado mayoritariamente es en la escuela, y este a su vez en el oficio maestro, llegando a formar parte de la cotidianidad escolar" (pág. 5). Con esta apreciación podemos comprender que la intrusión de las TIC en el ámbito educativo ha significado el avance significativo tanto para el alumno como para el docente. Las herramientas TIC cumplen un rol de facilitador entre la relación existente del alumno con la adquisición de conocimientos, además de facilitar la labor del maestro con sus herramientas.

No se conoce exactamente aún el grado de importancia que tiene en la educación el manejo de las TIC. No cabe duda que la tecnología juega un papel muy importante en el sistema educativo. La tecnología facilita el proceso de enseñanza ya sea virtualmente con el manejo de plataformas o utilizando aparatos como el proyector, la computadora, la calculadora, el internet, etc. No obstante, Vinueza- Vinueza y Simbaña-Gallardo (2017) afirma que "Ecuador presenta atrasos en el uso de las TIC y en infraestructura de comunicaciones, situación que afecta al desarrollo productivo nacional y a la creación de puestos de trabajo para los jóvenes que ingresan al mercado laboral" (pág. 7). Con el paso de los años, el acceso a herramientas informáticas como computadoras o internet ha ido incrementándose; sin embargo, existen zonas del país que no poseen acceso a estas como sucede con el internet. Esto sucede debido a la falta de infraestructura y a los niveles tecnológicos existentes en el país.

\subsection{Plataformas virtuales educativas}

El empleo de plataformas virtuales en el sistema educativo ha innovado no solo la organización de datos, si no también que se presenta como una ayuda pedagógica tanto para profesores, alumnos como padres de familia. Además, dichas plataformas tales como; Caroline, Moodle, etc. Facilitan la exposición de información que los docentes consideren importantes. Al respecto Taipe (2016) plantea que "los Entornos Virtuales de Aprendizaje tienen la gran ventaja de ser una herramienta para llevar nuevas opciones de innovación a dichos procesos" (pág. 3). Lo cual da a entender que las plataformas virtuales son de gran utilidad en los procesos educativos, además de esta manera se hace uso de las TIC en la educación, las herramientas informáticas se presentan de una manera innovadora gracias a la globalización y al desarrollo de la tecnología.

Las plataformas virtuales educativas trabajan de manera eficiente, por lo que dichas plataformas presentan numerosas aplicaciones para todo tipo actividad ya sea informativa, entrega de tareas

Licencia Creative Commons Atribución 4.0 Internacional (CC BY 4.0)

Revista Cátedra, 3(2), pp. 99-111, mayo-agosto 2020. e-ISSN: 2631-2875

https://doi.org/10.29166/10.29166/catedra.v3i2.2210 
o evaluativa, de manera que agilita la gestión educativa tanto a los profesores como a los estudiantes y a los involucrados en el sistema educativo. Al respecto,Sánchez (2016) dice "el término de Plataforma engloba un amplio rango de aplicaciones informáticas instaladas en un servidor cuya función es la de facilitar al profesorado la creación, administración, gestión y distribución de cursos a través de Internet" (pág. 218). Por lo que por medio de las plataformas virtuales se puede trabajar en tiempo real y comunicarse.

Así como existen numerosas ventajas de las plataformas virtuales educativas, también existen ciertas desventajas puesto que no todos los alumnos tienen acceso a internet, por lo tanto, representaría gastos económicos en centros de computación o transporte para acercarse a una biblioteca. A demás se necesita que los alumnos estén comprometidos con las actividades que se desarrollen, puesto que depende de la disposición de ellos para un trabajo con resultados efectivos.

Existen 3 tipos de plataformas virtuales, las cuales son utilizadas para diferentes tipos de trabajos. Cada uno de estos tres tipos tienen características diferentes y sus funciones son específicas para cada uno de ellos, las cuales se muestran a continuación:

- Plataformas Comerciales. - Son aquellas plataformas a la que se debe realizar un pago previo antes ser de utilizarlas y así poder acceder a la información. Este tipo de plataformas no permite a sus usuarios modificar el sistema. Al respecto, Sánchez (2009) dice "son herramientas por las que hay que pagar una cuota de instalación y/o mantenimiento" (pág. 220). Además de ser muy ágiles en el proceso educativo, el mantenimiento es rápido y eficiente, al igual que el servicio técnico que estos softwares ofrecen, lo que permite que el software sea cómodo y de agrado para los usuarios.

- Plataformas de Software Libre. - Este tipo de plataformas se encuentran de manera gratuita, es decir, no se necesita realizar algún pago para ingresar a la información. A demás permite realizar modificaciones en el sistema, Sánchez (2009) dice "En la mayoría de las ocasiones no hay que pagar por actualizaciones ni por número de licencias" (pág. 222). Por ende, se confirma que este tipo de plataformas brindan un servicio gratuito.

- Plataformas de Software Propio. - Las plataformas de software propio son desarrolladas para uso interno de una institución educativa; su propósito es satisfacer una necesidad específica para la comunidad institucional; por lo tanto, este tipo de plataformas no son para uso público ni comercial. Al respecto, Sánchez (2009) dice "no se suelen publicitar ni en ocasiones ofrecer a la comunidad de Internet por responder a situaciones educativas concretas" (pág. 220). Por lo tanto, este tipo de software se desarrolla para uso propio de la institución educativa.

\subsection{Plataformas virtuales y su importancia hoy en día}

Frente a la crisis sanitaria que se vive actualmente debido al COVID-19, se presentan diferentes inconvenientes a nivel mundial en diferentes ámbitos, tales como: económico, político, educativo, etc. Por lo tanto, hoy en día, existen diferentes plataformas creadas por instituciones privadas y públicas, las cuales contribuyen al desarrollo social, laboral y educativo. Según Cevallos et al. (2019) afirma que "actualmente un número considerable de organizaciones tanto del sector público como privado se encuentran promoviendo el diseño e implementación de herramientas virtuales informáticas y de objetos virtuales de aprendizaje" (pág. 69). Por ende, las plataformas virtuales son las principales herramientas utilizadas hoy en día con distintos propósitos, ya sea laborales, sociales o educativas como alternativa a la imposibilidad de asistir a trabajos, escuelas y universidades.

Sin embargo, las plataformas virtuales tienen tanto desventajas como ventajas. Existen usuarios que utilizan dichas plataformas para compartir información errónea, lo que ocasiona que otros

Licencia Creative Commons Atribución 4.0 Internacional (CC BY 4.0)

Revista Cátedra, 3(2), pp. 99-111, mayo-agosto 2020. e-ISSN: 2631-2875

https://doi.org/10.29166/10.29166/catedra.v3i2.2210 
usuarios se confundan. Al respecto, Cevallos y Villanueva (2019) enuncia que "se puede tomar en cuenta las amplias ventajas en el manejo digital, sin embargo, no se debe de perder en cuenta que, gracias al avance digital, de igual manera se encuentran usuarios que divulgan informaciones irrelevantes" (pág. 3). Por lo cual la plataforma virtual debe usar, con el fin de compartir información que sea de utilidad para el desempeño de diferentes funciones.

Existen diferentes ventajas que nos brindan las plataformas virtuales. El correcto uso de las herramientas virtuales actualmente, ayudan a la continuidad del trabajo y de los estudios, además de mantener comunicados a los diferentes usuarios, según Jaime (2019) enuncia:

Las páginas web son medios de comunicación que permiten al usuario que entra en la mismas, comunicarse con un contenido y también puede poner su opinión en un comentario y así interactuar con otras personas. Si no se tienen enciclopedias cerca y necesitamos buscar información instantánea sin adentrarnos al contenido, podemos buscar información en las páginas web, y esto es una gran ventaja ya que se tienen datos rápidos y fáciles de conseguir. Las personas pueden ingresar en cualquier momento y desde cualquier equipo (pág. 10).

Por lo que se puede decir que el correcto uso de las plataformas virtuales es de gran utilidad, ya sea en el ámbito social, económico, social y educativo, debido a que ayuda considerablemente al progreso de la sociedad.

De igual manera, el mal uso de las plataformas virtuales provoca confusiones y retrasos en trabajos y estudios, debido a que se puede transmitir información errónea o sin importancia, por lo que el compromiso de los usuarios también radica en ser responsables al momento de usarlas, de igual manera una desventaja significativa radica en el interés que presten los usuarios a dichas plataformas. Al respecto, Jaime (2019) expresa:

Cualquier persona del mundo puede subir información que no es cierta simplemente porque lo beneficia en algún sentido económico o social. El aislamiento que se puede llegar a dar entre seres humanos, eliminando la interacción social física. Requiere equipos y recursos técnicos. Los archivos se dejan guardados en el servidor de la institución u/o empresa que ofrecen herramientas Informáticas, quedando vulnerables a los usuarios. Puede convertirse en un arma de doble filo; se puede evidenciar si no se guía correctamente y si no se mantiene un control estricto en su uso (pág. 11).

De acuerdo a lo expresado, se puede afirmar que un mal uso de las herramientas tecnológicas puede provocar diferentes inconvenientes a la comunidad.

\subsection{Plataforma EducarEcuador}

Es un portal de servicios educativos que invita a la comunidad a ser parte de la automatización de servicios dentro de un proceso de mejora continua con el objetivo de brindar servicios de calidad a la ciudadanía y acercarse más a ella, mediante el uso de herramientas tecnológicas disponibles para toda la comunidad educativa.

Es el portal educativo que facilita el seguimiento y control de la gestión educativa para contribuir al mejoramiento continuo de la calidad de la educación, mediante la implementación de servicios virtuales como: gestión de control escolar, gestión docente, trámites ciudadanos, etc. Según el Ministerio de Educación (2009) dice: 
Educar Ecuador, orienta sus servicios educativos en línea a los diferentes actores de la comunidad educativa: estudiantes, docentes, autoridades de instituciones educativas, padres de familia y comunidad en general que forman parte del sistema educativo fiscal, particular, fiscomisional o municipal como es el servicio para el cobro de pensiones, matrículas y servicios educativos para instituciones educativas particulares y fiscales (pág. 1).

Por lo expresado anteriormente, el portal de servicios educativos virtuales Educar Ecuador, genera facilidades de acceso a la ciudadanía y satisface la demanda de una institución moderna que va a la vanguardia del uso de las tecnologías de la información.

\section{Trabajos relacionados}

En el año 2018, se realizó un estudio a cerca del manejo de la plataforma EducarEcuador a 132 profesores de la unidad educativa en la Unidad Educativa Santo Domingo de los Colorados, como resultados se evidenció que el $74 \%$ de los profesores conocían del funcionamiento de la plataforma virtual, mientras que el $26 \%$ no tenía conocimiento acerca del manejo de la plataforma. Por lo tanto, los autores concluyen que se deben realizar talleres de capacitaciones para aquellos profesores que no manejen de manera eficiente la plataforma virtual, para posteriormente evaluarlos nuevamente (Mora-Zambrano, Bonilla-Jurado, Núñez-Freirere y Sarmiento- Saavedra, 2018).

En el año 2018, se realizó un estudio de las dificultades de los docentes de la escuela "Dr. Leónidas García Ortiz" de Riobamba en la plataforma EducarEcuador. En los resultados se determina que en la institución educativa existen problemas en la manipulación de la plataforma EducarEcuador. Por lo tanto, concluye la autora que el problema se encuentra en la falta de conocimiento de las herramientas tecnológicas, que hace que el profesor se sienta inseguro para ejecutar actividades de organización y almacenamiento de información, de contenido y de datos en las plataformas digitales (Aliaga-Erazo, 2018).

En un estudio realizado en el año 2017, se diagnosticó el uso de la plataforma virtual EducarEcuador a 121 docentes de diferentes unidades educativas fiscales en la ciudad de Calceta. En los resultados presentados por el autor se da a conocer que, aproximadamente el $66.4 \%$ de los docentes maneja la plataforma eficientemente, mientras que, el 33.6\% no manejaría de manera correcta la plataforma. Por lo cual, la autora concluye que el número de horas que se proporciona a los docentes para el empleo eficiente de la plataforma no son suficientes (Lopez-Alava, 2017).

En el año 2016, en una investigación se estudió el uso de la plataforma EducarEcuador de las actividades educativas de los docentes en la unidad educativa Mitad del mundo. Los resultados arrojan un conjunto de deficiencias como: no existe un control acerca del cronograma establecido en la plataforma, los docentes no tienen el conocimiento adecuado para enviar o ubicar las tareas subidas por sus alumnos, inconvenientes suscitados en el ingreso del rendimiento académico, no poseen el conocimiento suficiente para el manejo de los paquetes ofimáticos que posee la plataforma (Barrionuevo-Murminacho, 2016).

En un estudio en el año 2016, se realizó una investigación a las instituciones educativas correspondientes a los cantones de Balsas, Marcabelí y Piñas en la provincia de El Oro, los que conforman el distrito 07D04 de Educación. Los resultados determinan que un pequeño número de docentes utilizan la plataforma educativa EducarEcuador, sobre todo por la falta de conocimiento y capacitación recibida para el uso de las herramientas didácticas en la labor de enseñanza. Permitió evidenciar la necesidad de un plan de capacitación orientada a la adquisición de los conocimientos, habilidades y destrezas en el uso de las herramientas didácticas de la 
plataforma educativa EducarEcuador para que puedan ser aplicadas en la labor docente (UrgilésParedes, 2016).

La investigación de Guapizaca (2016) tuvo como objetivo la evaluación del nivel de incidencia del uso del Portal Educativo EducarEcuador en el seguimiento y control académico a los estudiantes de la Unidad Educativa del Milenio Intercultural Bilingüe "Chibuleo". En esta investigación se estipula medir cuál es el nivel de incidencia del uso del Portal Educativo EducarEcuador de los padres de familia en el seguimiento y control académico. Según los resultados obtenidos después de realizada la encuesta a los padres de familia se determina que los padres en un porcentaje medio equivalente al 39\% y bajo en un 35\% no utilizan el Portal Educativo EducarEcuador. Por lo que el investigador propone realizar una guía para el uso del Portal Educativo EducarEcuador de los padres de familia para mejorar el seguimiento y control académico (Guapizaca- Jinde, 2016).

En el año 2016, en una investigación se indagó acera de las plataformas virtuales en el proceso de enseñanza aprendizaje de los estudiantes del curso de primero de bachillerato de la unidad educativa Atahualpa de la provincia de Tungurahua. Los resultados que arrojó establecieron que muy pocos docentes conocen o están familiarizados con la utilidad de una plataforma virtual; los estudiantes manifiestan que la relación enseñanza-aprendizaje mejoraría si se manejara una plataforma virtual, la cual actuaría como un eje transversal que ayudaría a mejorar su comprensión; los docentes afirman que utilizar una herramienta informática, como es la plataforma virtual, permitiría mejorar la relación docente alumno (León-Taipe, 2016).

En otra investigación en el año 2015, sobre el uso de la plataforma EducarEcuador por los docentes del Colegio Fiscal Ochoa León, ubicado en la ciudad de Pasaje. En los resultados el autor evidenció que los docentes se encuentran en un proceso de empleo de la plataforma, puesto que se presenta como una estrategia innovadora en los procesos educativos. El autor concluye que la plataforma EducarEcuador sirve como ayuda tanto para los docentes como para estudiantes y padres de familia en los distintos procesos educativos logrando así un cambio en la educación (Mejía-Herrera, 2015).

En el año 2012, una investigación realizó la evaluación del portal educativo EducarEcuador"de los docentes de la Unidad Educativa Consejo Provincial de Pichincha, y su incidencia en el planteamiento de una propuesta de reingeniería. De los resultados, se obtiene que el $25.46 \%$ de los docentes se encuentran satisfechos con la disponibilidad de recursos y contenidos. El 56.52\% de los docentes de la Unidad Educativa Consejo Provincial de Pichincha se encuentran poco satisfechos al usar los recursos del portal. En la reingeniería del portal se debe dar especial protagonismo a esta observación (Ochoa-Saeteros, 2012).

\section{Metodología}

A continuación, se describen los pasos utilizados en esta investigación:

1. Selección de bibliografía relacionada con el tema de investigación: se utilizó los repositorios con información acerca de la plataforma EducarEcuador, además las estadísticas presentadas sobre la plataforma.

2. Evaluación de resultados extraídos de las investigaciones: para determinar la accesibilidad de la plataforma se evaluaron las estadísticas y los documentos bibliográficos. Con esto se establecerá cuáles son las instituciones que más utilizan la plataforma. 
3. Nivel de factibilidad de la plataforma EducarEcuador: se analizó los resultados con el fin de establecer la calidad de funcionamiento y destreza de manejo de la plataforma por parte de la comunidad educativa.

\section{Resultados}

El Ministerio de Educación del Ecuador (2019), recoge datos de las instituciones públicas, privadas, fiscomisionales y municipales (estudiantes, docentes, instituciones) a nivel nacional y territorial al inicio y al final del año escolar. La información está disponible a partir del periodo 2009-2010 al 2018-2019. Con la información obtenida en el periodo 2018-2019 se determina que la plataforma se usa en 16200 instituciones educativas. Actualmente existen dos periodos educativos el régimen escolar Costa donde se encuentra comprendido Galápagos y el régimen escolar Sierra que abarca la región Amazónica. La región Costa se encuentra formada por 9484 instituciones, como se puede apreciar en el Cuadro 1. El régimen Sierra se encuentra formado por 6716 instituciones educativas, como se puede ver en el Cuadro 2.

\begin{tabular}{llllll}
\hline Área & Fiscal & Fiscomisional & Municipal & Particular & Total \\
\hline Rural & 3682 & 52 & 13 & 98 & 3845 \\
Urbana & 3908 & 131 & 30 & 1570 & 5639 \\
Total & 7590 & 183 & 43 & 1668 & 9484 \\
\hline
\end{tabular}

Cuadro 1. Régimen Costa. Fuente: (Ministerio de Educación, 2019, pág. 2)

\begin{tabular}{llllll}
\hline Área & Fiscal & Fiscomisional & Municipal & Particular & Total \\
\hline Rural & 3145 & 153 & 11 & 320 & 3629 \\
Urbana & 1674 & 216 & 61 & 1136 & 3087 \\
Total & 4819 & 369 & 72 & 1456 & $6716 \mathrm{~g}$ \\
\hline
\end{tabular}

Cuadro 2. Régimen Sierra. Fuente: (Ministerio de Educación, 2019, pág. 2)

\section{1. Índice de utilización de la plataforma EducarEcuador}

EducarEcuador es una plataforma netamente escolástica, es por esto que diversas instituciones educativas no hacen uso de todas sus herramientas, sin embargo, cabe recalcar que todas las instituciones educativas utilizan la plataforma EducarEcuador, puesto que la utilizan con el fin de realizar el proceso de titulación. Debido a esto las instituciones fiscales han tenido un aumento en registro anual en la plataforma, ver figura 1.

- Fiscal • Fiscomisional • Municipal • Particular

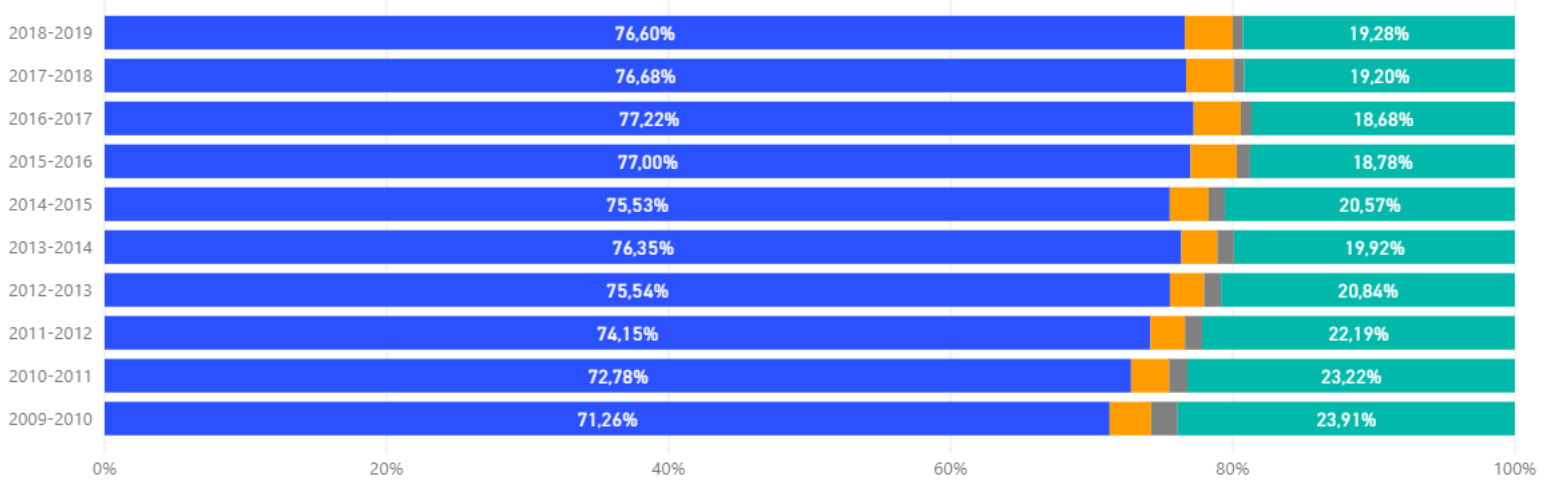

Figura 1. Por periodo y sostenimiento. Fuente: (Ministerio de Educación, 2019, pág. 1) 
En las unidades educativas fiscales y fiscomisionales, el índice de utilización ha incrementado desde el 2009 hasta el año 2019 puesto que en las unidades educativas fiscales ha incrementado $5.35 \%$ y las fiscomisionales en un $0.47 \%$ en la aceptación de la plataforma EducarEcuador. Mientras que en las unidades Educativas municipales ha decrecido el índice de aceptación en un $1.17 \%$; en las unidades educativas particulares ha llegado a decrecer en un $4.63 \%$. Por lo que se deduce que la plataforma educar ecuador no tiene un 100\% de aceptación a nivel nacional.

\subsection{Evaluación de la accesibilidad}

Con la finalidad de evaluar la accesibilidad de la plataforma EducarEcuador se utiliza un análisis bibliográfico, además de experiencias que fueron compartidas por diversas personas que se encuentran relacionadas con el sistema educativo. Según el último censo realizado por el INEC (2016) se establece que "el 36\% de los hogares a nivel nacional tienen acceso a internet, 13.5 puntos más que hace cinco años. En el área urbana el crecimiento es de 13.2 puntos, mientras que en el rural de 11,6 puntos" (pág. 7). Ecuador presenta un gran atraso en la infraestructura de tecnologías, información y comunicaciones. Uno de los indicadores más importantes es el acceso a internet en el cual hasta el año 2016 el país presenta tan solo un 36\% de cobertura a nivel nacional, ver figura 2 .

\section{Acceso al Internet según área}

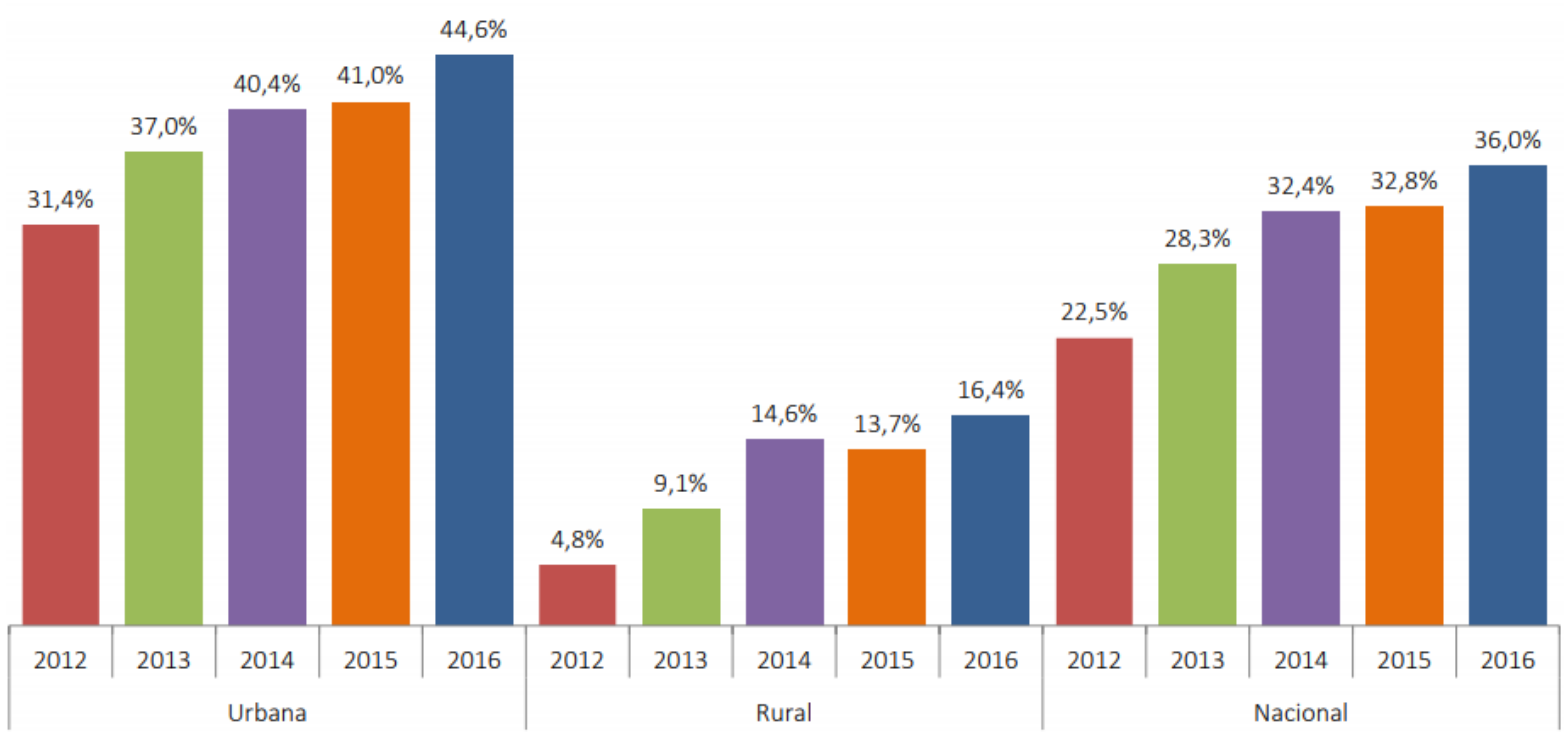

Figura 2. Acceso a internet según área. Fuente: (INEC, 2016, pág. 4)

De esta figura se puede corroborar las experiencias que afirman que es muy complicado que los estudiantes manejen la plataforma al 100\%, debido a que no todos los estudiantes poseen internet en sus hogares y mucho menos poseen un computador.

Existen diversos inconvenientes que dificultan la accesibilidad y el manejo de la plataforma, entre estos están el acceso a internet, el equipamiento del hogar he incluso la destreza de los usuarios de esta plataforma. No todas las personas poseen la capacidad del manejo de tecnología, incluyendo los docentes, ya que existen muchos maestros, que incluso son de edad avanzada, a los cuales se les dificulta mucho el manejo de la tecnología. También existen casos en que los docentes tienen que movilizarse de la comunidad en la que se encuentran para poder tener acceso a un computador con internet para así cargar las notas de sus estudiantes en la plataforma. Ahora 
bien, los inconvenientes son solamente de parte de los usuarios, y la infraestructura de TIC también repercute en problemas técnicos de la plataforma.

Los principales problemas registrados son el colapso de la plataforma por varios días y el incumplimiento de las fechas de habilitación de la plataforma para que los docentes puedan cargar las notas de los estudiantes, esto hasta una semana antes de la finalización del primer quimestre.

\section{Conclusiones}

Con los resultados expuestos en la presente investigación sobre la implementación de la plataforma educativa EducarEcuador, se demuestra que dicha plataforma ha tenido un gran índice de utilización por las instituciones educativas del Ecuador. Así, se ha podido constatar que la plataforma educativa está implementada en todas las unidades educativas a nivel nacional. Así también, contrastando con los resultados se puede decir que las instituciones educativas fiscomisionales, municipales y particulares también hacen uso de la plataforma educativa EducarEcuador, aumentando así el nivel de aceptación de la plataforma a nivel nacional. Esto se demuestra en el hecho que las unidades particulares se registran en la plataforma EducarEcuador únicamente para realizar el proceso de titulación de sus estudiantes y que éste sea validado por el Ministerio de Educación.

En las zonas rurales existen ciertas limitantes para acceder a la plataforma, ya sea por el difícil acceso al servicio de internet, lo que provoca que los usuarios tengan que movilizarse hacia otro lugar para acceder a la plataforma; además, la falta de manejo de tecnología y de acceso a la misma en las zonas rurales, causan que los profesores y la comunidad no se familiaricen con las herramientas informáticas, los cuales son los principales problemas para que los educadores no manejen la plataforma EducarEcuador de una manera óptima; de igual forma, el problema de manejo de la plataforma es aún mayor en docentes de edad avanzada, puesto que se les dificulta el manejo no sólo de la plataforma EducarEcuador, sino las herramientas informáticas que hoy en día son tan utilizadas.

Esta investigación comprueba lo indispensable de la tecnología en el ámbito de la educación en el Ecuador. Además, se ha podido constatar que la plataforma educativa EducarEcuador es una herramienta de gran ayuda en el sistema educativo. Sin embargo, esta herramienta no es totalmente aprovechada por los docentes y estudiantes por factores como las limitaciones al acceso de internet y el desconocimiento de los docentes en el uso de la plataforma EducarEcuador. Por los factores expuestos anteriormente se puede afirmar que el sistema educativo ecuatoriano no cuenta con el avance que debería en el ámbito tecnológico. La importancia que posee hoy en día el uso de plataformas virtuales, se ve reflejada en la necesidad que tienen las diferentes instituciones, tanto públicas como privadas, en la producción de trabajo mediante reuniones virtuales y el teletrabajo, y en el campo educativo, puesto que diversas instituciones educativas se han visto obligadas a utilizar herramientas educativas, como son las plataformas virtuales educativas con el propósito de reforzar y mantener la continuidad académica.

\section{Bibliografía}

Aliaga-Erazo, A. D. (2018). Estudio de las dificultades de los docentes de la escuela "Dr. Leónidas García Ortiz" de Riobamba en la plataforma EducarEcuador. Pontificia Universidad Católica del Ecuador, Quito. 
Barrionuevo-Murminacho, E. B. (2016). Uso de la plataforma de gestión de servicios educativos EducarEcuador en las actividades educativas de los y las docentes de la unidad educativa "Mitad del mundo". Quito.

Cabrera-Almenara, J., y LLorete-Seguro, M. (2005). Las plataformas virtuales en el ámbito de la teleformación. España.

Cevallos, D., y Villanueva, J. (2019). Influencia de las plataformas virtuales radiofónicas y su evolución ante la construcción de nuevos formatos informativos. Caso de estudio Wilar RTV y WQ Radio. Revista Ceribeña de Ciencias Sociales.

Cevallos, O. I., Mejía, L. A., y Arango-Medina, D. (2019). Auditoria de usabilidad de herramientas implementadas en las plataformas virtuales para ofertar servicios con responsabilidad social. revista Innova Itfip, 69.

Guapizaca -Jinde, A. P. (2016). Uso del portal educativo EducarEcuador de los padres de familia en el seguimiento y control académico a los estudiantes de la unidad educativa del milenio intercultural bilingüe "Chibuleo" de la comunidad de Chibuleo san francisco. Ambato.

Hernández, R. M. (2017). Impacto de las TIC en la educación: Retos y Perspectivas. Lima-Peru: Universidad San Ignacio de Loyola.

INEC. (2016). Tecnologías de la Información y Comunicaciones (TIC'S) 2016.

Jaime-Torres, X. A. (2019). Páginas web educativas. Recuperado el 01 abril de 2020, de http://repositorio.une.edu.pe/bitstream/handle/UNE/3986/MONOGRAF\%c3\%8dA\%2 0-\%20JAIME\%20TORRES.pdf?sequence=1\&isAllowed=y

López-Álava, J. V. (2017). Uso de la plataforma virtual "EducarEcuador" por parte de los docentes de las instituciones educativas fiscales de Calceta, cantón Bolívar. Calceta.

Mejia-Herrera, V. H. (2015). Uso de la plataforma virtual EducarEcuador como una estrategia innovadora en los procesos educativos del colegio fiscal Ochoa Leon. Machala.

Ministerio de Educación. (2016). Instituciones educativas deben registrar las calificaciones y asistencia de los estudiantes en Educar Ecuador. Recuperado el 02 de abril de 2020 de educacion.gob.ec: https://educacion.gob.ec/instituciones-educativas-deben-registrarlas-calificaciones-y-asistencia-de-los-estudiantes-en-educar-ecuador/

Ministerio de Educación. (2019). Vizualizador Estadistico. Instituciones 2018-2019. Quito.

Ministerio de Educación. (2009). EducarEcuador. Recuperado el 02 de abril de 2020, de educarecuador.gob.ec: https://educarecuador.gob.ec/que-es-educar-ecuador/

Mora -Zambrano, E. R., Bonilla-Jurado, D. M., Núñez -Freire, L. A., y Sarmiento-Saavedra, J. C. (2018). Inadaptabilidad de los docentes al manejo de plataformas virtuales: caso EducarEcuador. Revista Conrado.

Ochoa-Saeteros, A. C. (2012). El uso del portal educativo EducarEcuador de los docentes de la unidad educativa consejo provincial de pichincha y su incidencia en el planteamiento de una propuesta de reingeniería. Ambato.

Sánchez, J. (2009). Plataformas de enseñanza virtual para entornos educativos. Cevilla-España: Pixel-Bit. 
Taipe, L., y Araceli, V. (2016). Plataformas virtuales y proceso enseñanza aprendizaje en los estudiantes de primero de bachillerato de la unidad educativa Atahualpa de la parroquia Atahualpa del cantón Ambato provincia de Tungurahua. Ambato.

Urgilés-Paredes, C. A. (2016). Incidencia del uso de las herramientas didacticas de la plataforma EducarEcuador en la labor docente. Guayaquil.

Vinueza-Vinueza, S. , y Simbaña-Gallardo, V. (2017). Impacto de las TIC en la Educación Superior en el Ecuador. Revista Publicando, 4, 355-368. https://revistapublicando.org/revista/index.php/crv/article/view/530

\section{Autores}

SANTIAGO VINUEZA-VINUEZA obtuvo su título de Magister en Redes de Comunicaciones, de la Facultad de Ingeniería, de la Pontificia Universidad Católica del Ecuador en 2016, Magíster en Sistemas Informáticos Educativos, Universidad Tecnológica Israel en 2009, Licenciado en Ciencias de la Educación especialización de Informática, Facultad de Filosofía Letras y Ciencias de la Educación, Universidad Central del Ecuador en 2002, Ingeniero en Ejecución Informática, Universidad Autónoma de Quito en 2002.

Actualmente es profesor Agregado de la Facultad de Ciencias Económicas de la Universidad del Ecuador. Vocal Principal del Fondo de Cesantía de la Universidad Central del Ecuador, Vocal del H.C. Directivo de la Facultad de Ciencias Económicas de la Universidad Central del Ecuador, Coordinador de la Unidad de Titulación de la Carrera de Finanzas de la Universidad Central del Ecuador, Coordinador de la Asignatura de Sistemas de Información de la Facultad de Ciencias Económicas de la Universidad Central del Ecuador, Sus principales investigaciones se enmarcan en el campo educativo y las Tecnologías de la Información y Comunicación.

JOSHUA POZO-CAICEDO Actualmente es estudiante de tercer semestre de la carrera de Pedagogía de las Ciencias Experimentales Informática, de la facultad de Filosofía, letras y ciencias de la educación, de la Universidad Central del Ecuador. Estudiante de tercer nivel de la Suficiencia de Inglés en el Centro de Idiomas de la Universidad Central del Ecuador.

ANTHONY PACHECO-GALLEGOS Actualmente es estudiante de tercer semestre de la carrera de Pedagogía de las Ciencias Experimentales Informática en la Facultad de Filosofía, Letras y Ciencias de la Educación, de la Universidad Central del Ecuador. Estudiante de segundo nivel de la Suficiencia de Inglés en el Centro de Idiomas de la Universidad Central del Ecuador.

RICHARD AREQUIPA-CAISALUISA Actualmente es estudiante de tercer semestre de la carrera de Pedagogía de las Ciencias Experimentales Informática en la Facultad de Filosofía, Letras y Ciencias de la Educación, de la Universidad Central del Ecuador. 